\title{
Investigation of the mechanical properties of a low-shrinkage liquid crystalline matrix combined with nano-hydroxyapatite
}

This article was published in the following Dove Press journal:

International Journal of Nanomedicine

24 August 201I

Number of times this article has been viewed

\section{Wen Wen Liu' \\ Xiao Ping $\mathrm{He}^{1}$ \\ An Chun $\mathrm{Mo}^{2}$ \\ Qian Qian Yao' \\ Jun $\mathrm{Ye}^{1}$ \\ Nie Jing ${ }^{2}$}

'State Key Laboratory of

Oral Diseases, ${ }^{2}$ Department of

Oral Implant, West China College

of Stomatology, Sichuan University,

Chengdu, People's Republic of China

Correspondence: An Chun Mo

No 14, 3rd section, South Renmin Road, Chengdu, 61004I, People's Republic

of China

Tel +86 28-88550357I

Fax $+8628-85582167$

Email moanchun@I63.com
Abstract: The objective of this study was to investigate the mechanical properties of a lowshrinkage acrylate liquid crystalline resin matrix (ALCRM), (4-3-(acryloyloxy)-2-hydroxypropoxy) phenyl 4-(3-(acryloyloxy)-2-hydroxypropoxy) benzoate, combined with nano-hydroxyapatite (na-HA). The ALCRM monomer and diluent monomer triethylene glycol dimethacrylate (TEGDMA) were mixed at a mass ratio of 7:3 to prepare the resin matrix. The volume shrinkage of the ALCRM/TEGDMA and the traditional resin matrix Bis-GMA (2, 2-bis [4-(3-methacryloxy2-hydroxypropoxy) phenyl] propane)/TEGDMA were measured. Then $20 \%, 30 \%$, and $40 \%$ na-HA were added to ALCRM/TEGDMA and Bis-GMA/TEGDMA, respectively. Compressive strength (CS) and Vickers hardness (VHN) were tested to identify mechanical properties of the composites. The volume shrinkage of the ALCRM/TEGDMA was 3.60\% $\pm 0.36 \%$, which was lower than that of the traditional resin matrix. Moreover, with the same amount of na-HA filler, the mechanical properties of the ALCRM-based composites were superior to those of Bis-GMA-based composites. ALCRM-based composites exhibited the highest CS and VHN values when the filler content was $30 \%$. These results indicate that the low-shrinkage liquid crystalline resin matrix has strong mechanical properties after incorporating the na-HA. The experimental ALCRM-based composites have promising potential for the development of novel low-shrinkage dental resins with strong mechanical properties.

Keywords: liquid crystalline matrix, nano-hydroxyapatite, compressive strength, Vickers hardness

\section{Introduction}

Resin composites have been widely used in esthetic restorative treatments. Their characteristics, such as manipulative quality, durability, and wear resistance, have been improved markedly. ${ }^{1}$ However, the currently used resins in composite systems have some shortcomings that limit their applications, such as polymerization shrinkage and low mechanical properties. ${ }^{2}$ Scientists have developed some matrices with lower polymerization shrinkage. ${ }^{3-6}$ Compared with the traditional acrylic resin, liquid crystal polymers (LCPs) have become the focus for their unique structures and mechanical properties. Researchers at the University of Texas Health Science Center developed a new kind of liquid crystalline matrix with remarkably low polymerization shrinkage. However, the mechanical properties of composites based on liquid crystalline matrices still need more investigation.

Dental resin composites are composed of a resin matrix (continuous phase) and inorganic fillers (dispersed phase). The resin matrix is the primary component of dental composites. Incorporation of inorganic fillers is an effective way to improve the 
mechanical properties of resin matrices. The content and size of inorganic fillers also play an important role in the properties of resin composites. ${ }^{7}$ In addition, fillers can reduce the thermal expansion coefficient and the volume shrinkage of resin composites during polymerization. ${ }^{8}$ Resin composites containing nano-fillers have excellent properties such as better application abilities and aesthetics, and higher strength and wear resistance. ${ }^{8}$ Hydroxyapatite has excellent biological compatibility and good physical properties. Apatite particles can form strong active connections with bones and improve the remineralization of teeth. Since hydroxyapatite is an adsorbent of proteins and enzymes, it can block cracks to prevent dental caries and repair the defects on the tooth surface. ${ }^{9}$ In fact, the human tooth is a kind of composite composed of nano-apatite crystals and collagen matrix. ${ }^{5}$ Previous animal experiments demonstrated that nano-hydroxyapatite (na-HA) can promote the formation of osteodentin by aggregation of odontoblasts and increasing the activity of alkaline phosphatase and lactate dehydrogenase enzyme. ${ }^{10}$ Therefore, using na-HA as a filler to synthesize the nano-composites has attracted the attention of dentists and materials scientists.

In this research, a liquid crystalline resin matrix (ALCRM), (4-3-(acryloyloxy)-2-hydroxypropoxy) phenyl 4-(3(acryloyloxy)-2-hydroxypropoxy) benzoate, was developed and characterized with low polymerization shrinkage. An investigation of the mechanical properties of the low-shrinkage ALCRM-based composites which incorporated various proportions of nano-fillers was carried out. Using triethylene glycol dimethacrylate (TEGDMA) as the diluent monomer, the compressive strength (CS) and Vickers hardness (VHN) of the experimental composites (ALCRM/TEGDMA/na-HA) and the control composites 2, 2-bis [4-(3-methacryloxy-2hydroxypropoxy) phenyl] propane ([Bis-GMA]/TEGDMA/ na-HA) were determined. The results of the study show that ALCRM/TEGDMA/na-HA has strong mechanical properties which shows their potential for the development of a lowshrinkage liquid crystalline nano-composite resin.

\section{Materials and methods Materials}

Hydroquinone (DHP), 4-hydroxybenzoic acid(HBA), boric acid $\left(\mathrm{H}_{3} \mathrm{BO}_{3}\right)$, sulfuric acid $\left(\mathrm{H}_{2} \mathrm{SO}_{4}\right)$, toluene $(\mathrm{MB})$, p-xylene (DMB), 2-(chloromethyl) oxirane (ECH), N-butyl-N,N-dipropylbutan1-aminium bromide (TBAB), acrylic acid (AA) and N,Ndimethylbenzenamine (DEBA) were purchased from Xi'an Chemical Reagent Factory, China. Bis-GMA was used as resin matrix (Sigma-Aldrich, St Louis, MO), TEGDMA (Wako and Guang Chun Le Co Japan) was used as diluent, camphorquinone
(CQ) (Alfa Aesar Co, Ward Hill, MA) and 2-(dimethylamino) ethyl methacrylate 2 (DMAEMA) (Sigma-Aldrich) were used as photosentizer (Alfa Aesar Co USA), na-HA crystals (Guona Technology Co, Ltd China) was used as inorganic filler for the resin composites, butylated hydroxytoluene (BHT) was used as inhibiter (Shanghai Reagent Factory, China); 3-methacryloyloxy propyl trimethoxy silane (KH-570) was purchased from Chenguang Coupling Agent Co, Ltd China.

\section{Instruments}

CS was measured by L-500 tensile testing machine; VHN was determined by HBRV-1857.5 Brinell Hardness Tester.

\section{Synthesis of ALCRM}

HBA (4.87 g) and DHP (5 g) (molar ratio of 1:1.1) were added to a $250-\mathrm{mL}$ three-neck flask. Then boric acid $(0.1733 \mathrm{~g})$, sulfuric acid $(0.25 \mathrm{~mL})$, toluene $(20 \mathrm{~mL})$, and DMB $(5 \mathrm{~mL})$ were added to the flask. The mixture was stirred until a homogeneous liquid was obtained. Then the mixture was heated under nitrogen atmosphere to obtain the liquid crystalline unit: mesogenic unit (4-hydroxyphenyl-4-hydroxybenzoate [HPHB]). HPHB (5.66 g), ECH (50 mL excess 15 times) and TBAB $(0.12 \mathrm{~g})$ were loaded into a one-neck flask followed by $30 \mathrm{~mL}$ of methanol. After series of reactions, the epoxy compound (4-(oxiran-2-ylmethoxy)-phenyl4-(oxiran-2ylmethoxy) benzoate [EPEB]) was prepared. EPEB (3.63 g) and the inhibitor DHP $(0.01 \mathrm{~g})$ were loaded into a three-neck flask. Meanwhile, AA (1.93 g, EPEB/AA molar ratio was $1: 2.2)$ was weighed into a beaker, followed by the addition of DEBA $(0.05 \mathrm{~mL})$. The product obtained was ALCRM.

\section{Determination of the volume shrinkage}

The ALCRM and TEGDMA were mixed at the mass ratio of 70:30 as the experimental group. The photosensitive initiating system camphorquinone (CQ $1 \mathrm{wt} \%$ )/2-(dimethylamino) ethyl methacrylate (DMAEMA $2 \mathrm{wt} \%$ ) was added to the mixture and homogenized. The polymerization shrinkage of the material was determined with an expansimeter using the method of Rees et al. ${ }^{11}$ The volume shrinkage was calculated by the volume change before and after curing. Each group was tested three times at room temperature. The result was calculated from the arithmetical mean $(\bar{X})$ and standard deviation of one group.

The equation is as follows:

Rate of volume shrinkage $=\Delta V / V_{0} \times 100 \%$

$V_{0}$ : volume of the uncured material $(0.5 \mathrm{~mL})$.

$\Delta V$ : volume change $(\mathrm{mL})$. 
The Bis-GMA in the control group was tested by the same method.

\section{Coupling treatment for the surface of na-HA}

The mixture of 3-(methacryloyloxy) propyl trimethoxy silane and the ethanol aqueous solution $(\mathrm{pH}=3.5-5.0,25 \mathrm{wt} \%)$ was added to na-HA under ultrasonic vibration. After coupling treatment for 30 minutes at $50^{\circ} \mathrm{C}$, the na-HA was washed with deionized water and dried for about 3 hours at $80^{\circ} \mathrm{C}$ untill the filler was dried completely. Lastly the filler was dried at $105^{\circ} \mathrm{C}$ for 30 minutes.

\section{Preparation of composites}

The filler of na-HA and resin matrix (ALCRM/TEGDMA 7:3) were mixed at mass ratios of 20:80,30:70, and 40:60 to prepare three corresponding groups of resin composites I, II, and III.

With the same methods, the filler of na-HA and Bis-GMA/TEGDMA (7:3) were also mixed at ratios of 20:80, 30:70, and 40:60 to prepare the three groups of resin composites IV, V, and VI.

\section{Determination of compressive strength and Vickers hardness of composites}

The diameters and heights of cured resin composites (I-VI) samples were measured and preserved in water at $37^{\circ} \mathrm{C}$ for 24 hours. Then the CSs of samples were measured at a compression speed of $10 \mathrm{~mm} / \mathrm{min}$ with a L-500 tensile testing machine. The maximum load $P(\mathrm{~N})$ was recorded when the sample was destroyed. The compressive strength was calculated by following equation (2):

$$
\mathrm{CS}=P / r^{2}(\mathrm{MPa})
$$

$P$ : maximum load

$r$ : radius of the specimen

VHN of the cured samples (I-VI) was measured with load (30 kgf) by a Brinell Hardness Tester (HBRV-1857.5).

Table I Polymerization shrinkage of ALCRM and Bis-GMA with TEGDMA 7:3 in light cure

\begin{tabular}{lll}
\hline Samples & ALCRM/TEGDMA (\%) & Bis-GMA/TEGDMA (\%) \\
\hline $\mathrm{I}$ & 4.0 & 6.3 \\
2 & 3.5 & 5.7 \\
3 & 3.3 & 5.5 \\
$\chi \pm \mathrm{SD}^{\mathrm{a}}$ & $3.60 \pm 0.36$ & $5.83 \pm 0.42$ \\
\hline
\end{tabular}

Note: a Data presented as mean and standard deviation $(\chi \pm S D)$, and $\mathrm{n}=3$. Abbreviations: ALCRM, acrylate liquid crystalline resin matrix; Bis-GMA, 2, 2-bis [4-(3-methacryloxy-2-hydroxypropoxy) phenyl] propane; TEGDMA, triethylene glycol dimethacrylate; SD, standard deviation.
The diagonal lengths of the indentations on samples were measured by the reading microscope and then the hardness values were obtained. The results of CS and VHN were calculated from the arithmetical means $(\bar{X})$ and standard deviations of one group that contained five samples.

\section{Results and discussion}

Comparing ALCRM wotj Bis-GMA by the Student-NewmanKeuls statistical method revealed that ALCRM exhibited a lower volume shrinkage rate (Table 1). The same method showed that ALCRM/TEGDMA/na-HA exhibited a higher $\mathrm{CS}$ and VHN (Figure 1A and B). All the differences were statistically significant $(P<0.05)$. With the same content of filler, the mechanical properties of ALCRM/TEGDMA/ na-HA were better than those of Bis-GMA/TEGDMA/ na-HA. The strong properties of ALCRM are attributed to the
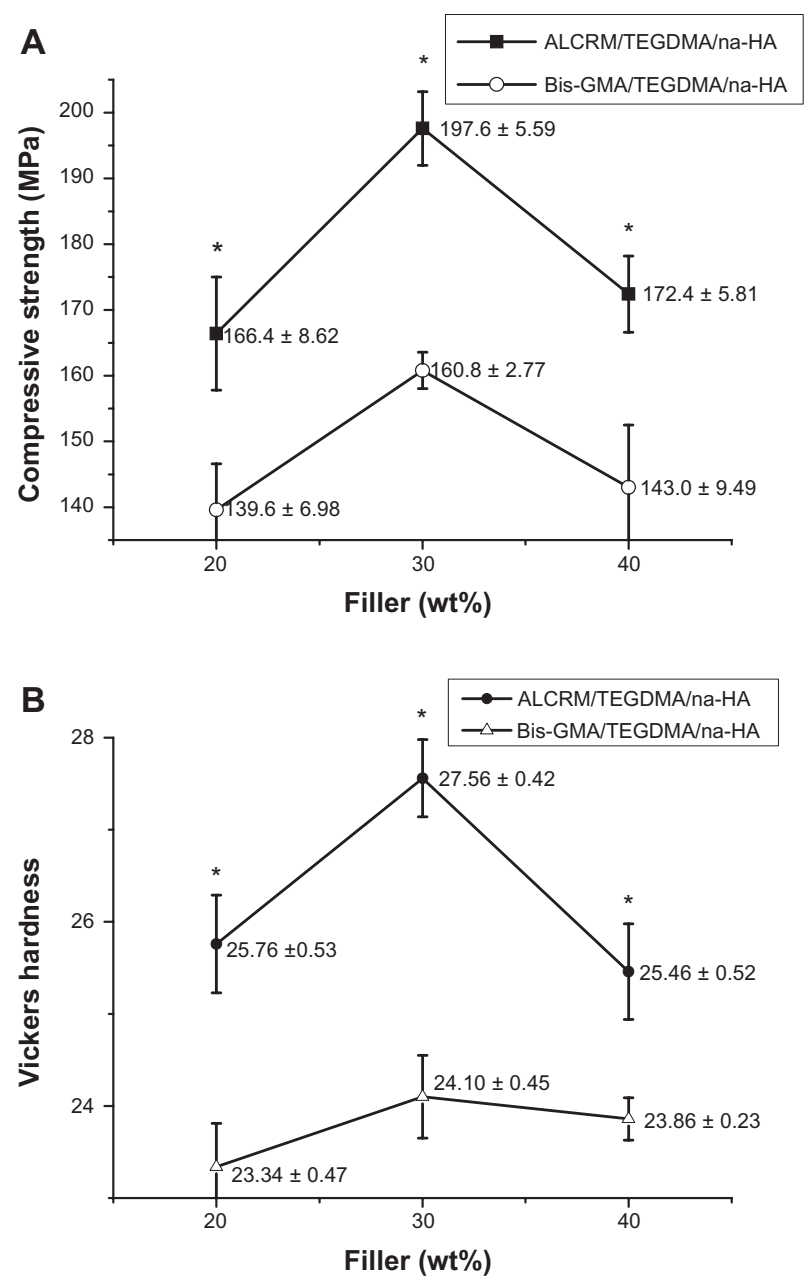

Figure I (A) Compressive strengths and (B) Vickers hardness of ALCRM/TEGDMA and Bis-GMA/TEGDMA with different proportions of na-HA filler.

Notes: Data are presented as mean and standard deviation $(\chi \pm S D)$, and $n=5 ; * P<0.05$. Abbreviations: ALCRM, acrylate liquid crystalline resin matrix; Bis-GMA, 2, 2-bis [4-(3-methacryloxy-2-hydroxypropoxy) phenyl] propane; na-HA, nano-hydroxyapatite; TEGDMA, triethylene glycol dimethacrylate; SD, standard deviation. 
mesogenic unit. The existence of the mesogenic unit is the key factor for the matrix to exhibit liquid crystalline properties. The mesogenic unit is composed of rod-like molecules in an ordered layered arrangement (Figure 2A); the order of the molecular structure is disarranged to form a network during polymerization (Figure 2B). As long as the liquid crystalline matrix is polymerized in its mesophase range, it retains all of its optical and mechanical properties. (This phenomenon is called the "memory effect" of liquid crystalline polymers. ${ }^{12-14}$ ) These rigid rod-like molecular structures of polymers have strong interactions with each other. Two of the most important interactions are resonance and steric hindrance. Resonance takes place in the ALCRM, which is an aromatic compound. Around the covalent compounds, the electron clouds distribute uniformly and strengthen the interface of the polymer. In addition, the rigid rod-like molecules that disperse among the polymer chains will reinforce the composites. ${ }^{15}$ These phenomena that were generated during curing to enhance the mechanical properties of the resin composites are called self-reinforcement of the mesogenic unit. ${ }^{16}$

Of the ALCRM-based composites I, II, III, the CS and VHN of composite II was higher than that of I and II $(P<0.05)$ (Figure $1 \mathrm{~A}$ and $\mathrm{B})$.

In the development of the mechanical properties of ALCRM-based composites, particle size and content of filler are important factors as well as the matrix. The filler used in this study was na-HA $(d=20-30 \mathrm{~nm})$, which has a structure similar to that of human bones and teeth. Using na-HA as a filler could reinforce the rod-like structures of mesogenic unit and improve the mechanical properties of ALCRM (Figure 2C).

In order to obtain excellent mechanical properties, resin composites should contain as much inorganic fillers as possible. At present, inorganic fillers usually contain 35\%-90\% (mass fraction). The increase of filler content could improve hardness, strength, and wear resistance of polymers. ${ }^{17}$ However, the filler content of composites can be reduced due to their finer particle size and higher surface activity. ${ }^{18}$ The nano-filler is difficult to load and disperse in a high proportion of resin matrix. The particle size distribution is a key factor for the content of the filler in resin matrix. Wide size distributions can reduce the gaps between the filler particles and increase the content of filler.

As the analysis above shows, with the same na-HA content, ALCRM-based composites showed higher mechanical properties than the Bis-GMA-based composites. The mesogenic unit contributed to the strong mechanical properties of ALCRMbased composites. When using ALCRM/TEGDMA as the resin matrix, composite II performed best. The contents
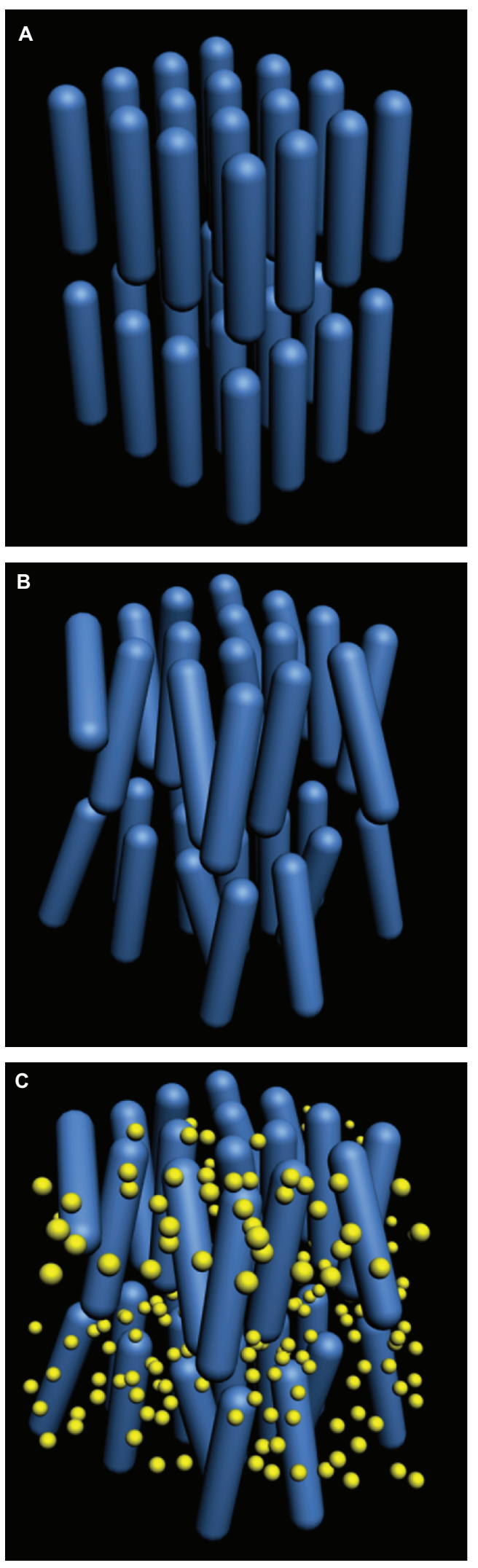

Figure 2 (A) The rod-like molecules of mesogenic unit of ALCRM and (B) the arrangement of the rod-like molecules during polymerization; (C) na-HA filler (yellow sphere) reinforce the rod-like structure.

Abbreviations: ALCRM, acrylate liquid crystalline resin matrix; na-HA, nanohydroxyapatite. 
of nano-filler in matrix have a maximum value. Below the maximum value, the performance of composites will be enhanced as the filler content level increases. Beyond the maximum value, excessive filler content will reduce the properties of the composites. ${ }^{19}$ This study demonstrates that the suitable mass ratio of na-HA and ALCRM/TEGDMA is 30:70.

\section{Conclusion}

Compared with Bis-GMA/TEGDMA, ALCRM/TEGDMAbased composites showed higher CS and VHN values with the same filler content. Using ALCRM/TEGDMA as resin matrix, the composite which contained $30 \mathrm{wt} \%$ na-HA fillers had optimum mechanical properties.

The low-shrinkage liquid crystalline matrix has strong mechanical properties when combined with na-HA filler. The mechanical properties could be improved by improving the methods of filler preparation. ${ }^{20}$ Improving the surface treatment and loading mode of the na-HA filler warrant further investigation. Moreover, due to the excellent biocompatibility of the na-HA, the higher the HA content, the better will be the bioactivity of the composites. ${ }^{21}$ Further experiments will also focus on the bioactivity of the composites and find the ideal proportions of the various components of this composites system. In a summary, this low shrinkage-liquid crystalline nano-composite exhibits strong mechanical properties and has promising potential for the development of novel superior dental resins.

\section{Acknowledgments}

This study was supported by funding from the Key Technologies Program, Sichuan, China (0040305301101). Thanks to the State Key Laboratory of Oral Diseases and Laboratory of liquid crystal polymer, Sichuan University.

\section{Disclosure}

The authors declare no conflicts of interest.

\section{References}

1. Kim JW, Kim LU, Kim CK, et al. Characteristics of novel dental composites containing 2, 2-Bis [4-(2-methoxy-3-methacryloyloxypropoxy) phenyl] propane as a base resin. Biomacromolecules. 2006;7:154-160.

2. Satsangi N, Rawls HR, Norling BK. Synthesis of low- shrinkage polymerizable liquid-crystal monomers. Biomed Mater Res B Appl Biomater. 2004; $71: 153-158$

International Journal of Nanomedicine

\section{Publish your work in this journal}

The International Journal of Nanomedicine is an international, peerreviewed journal focusing on the application of nanotechnology in diagnostics, therapeutics, and drug delivery systems throughou the biomedical field. This journal is indexed on PubMed Central, MedLine, CAS, SciSearch ${ }^{\circledR}$, Current Contents ${ }^{\circledR} /$ Clinical Medicine,
3. Kim LU, Kim JW, Kim CK. Effects of molecular structure of the resins on the volumetric shrinkage and the mechanical strength of dental restorative composites. Biomacromolecules. 2006;7:2680-2687.

4. Eva K Viljanen, Mikael Skrifvars, Pekka K Vallittu. Dendritic copolymers and particulate filler composites for dental applications: degree of conversion and thermal properties. Dent Mater. 2007;23:1420-1427.

5. Mohammad A, Mehdi A, Samal B, David CW. Synthesis, characterization, shrinkage and curing kinetics of a new low-shrinkage urethane dimethacrylate monomer for dental applications. Dent Mater. 2007;23:1030-1041.

6. Satsangi N, Rawls HR, Norling BK. Synthesis of low-shrinkage polymeriziable methacrylate liquid-crystal monomers. Biomed Mater Res B Appl Biomater. 2005;74:706-711.

7. Urabe H, Nomura Y, Shirai K, Yoshioka M, Shintani H. Effect of filler content and size to properties of composite resins on microwave curing. J Mater Sci Mater M. 1999;10:375-378.

8. Halperin WP. Quantum size effects in metal particle. Rev Mod Phys. 1986;58:532-539.

9. Xuejiang W, Yubao L, Jie W, Klass G. Development of biomimetic nano-hydroxyapatite/poly (hexamethylene adipamide) composites. Biomaterials. 2002;23:4787-4791.

10. Li T, Akao M, Takagi M. Tissue reaction of hydroxyapatite sol to rat molar pulp. J Mater Sci Mater Med. 1998;9:611-672.

11. Lee JY, Shim MJ, Lee HK, Kim SW. Reaction kinetics of liquid crystalline epoxy cured with aromatic diamine: its synthesis and mechanical and electrical characteristics. Appl Polym Sci. 2001;82: 2372-2380.

12. Corvazier L, Zhao Y. Induction of liquid crystal orientation through azobenzene containing polymer networks. Macromolecules. 1999;32: $3195-3200$.

13. Kurihara S, Sakamoto A, Nonaka T. Liquid crystalline polymer networks: effect of crosslinking on the stability macroscopic molecular orientation. Macromolecules. 1999;32:3150-3153.

14. Kurihara S, Sakamoto A, Yoneyama D. Photochemical switching behavior of liquid crystalline epoxy cured with aromatic diamine: its synthesis and mechanical and electrical characteristics. Appl Polym Sci. 2001;82:2372-2380.

15. Susheel K, Kaith BS, Sanjeev S, Bandna B. Mechanical properties of flax-g-poly(methyl acrylate) reinforced phenolic composites. Fiber Polym. 2008;9:416-422.

16. Amendola E, Giamberini M, Carfagna C. Self-toughing liquid crystalline vinyl ester adhesives. Macromol Symp. 2002;180:153-167.

17. Faltermeier A, Rosentritt M, Reicheneder C, Mussiq D. Experimental composite brackets influence of filler level on the mechanical properties. Am J Orthod Dentofacial Orthop. 2006;130:699.

18. Zhou YX, White E, M Hosur M, Jeelan S. Effect of particle size and weight fraction on the flexural strength and failure mode of $\mathrm{TiO} 2$ particles reinforced epoxy. Mater Lett. 2010;64:806-806.

19. Wang L, Weng L, Song SH, Sun QJ. Mechanical properties and microstructure of polyetheretherketone-hydroxyapatite nanocomposite materials. Mater Lett. 2010;64:2201-2204.

20. Sanosh KP, Min-cheol C, Balakrishnan A, Kim TN, Seong-Jai C. Preparation and characterization of nano-hydroxyapatite powder using sol-gel technique. Bull Mater Sci. 2009;32:465-470.

21. Jie W, Yubao L, Yi H. Processing properties of nanoapatite-polyamide biocomposites. J Mater Sci. 2005;40:793-796.

\section{Dovepress}

Journal Citation Reports/Science Edition, EMBase, Scopus and the Elsevier Bibliographic databases. The manuscript management system is completely online and includes a very quick and fair peer-review system, which is all easy to use. Visit http://www.dovepress.com/ testimonials.php to read real quotes from published authors. 